\title{
On The Capacity Of Multi-Antenna Gaussian Channels ${ }^{1}$
}

\author{
Nicolae Chiurtu, Bixio Rimoldi and Emre Telatar \\ Communication Systems Department \\ Swiss Federal Institute of Technology, Lausanne, Switzerland \\ e-mail: \{Nicolae.Chiurtu, Bixio.Rimoldi, Emre.Telatar\}@epfl.ch
}

\section{INTRODUCTION}

In this paper we investigate the use of multi-antennas at both ends of a point-to-point communication system over the additive Gaussian channel. We consider a system with $t$ transmit antennas and $r$ receive antennas in which the received vector $\mathbf{v} \in \mathbb{C}^{r}$ depends on the transmitted vector $\mathbf{u} \in \mathbb{C}^{t}$ via:

$$
\mathbf{v}=H \mathbf{u}+\mathbf{w}
$$

where $H \in \mathbb{C}^{\times t}$ is the channel transfer matrix and $\mathbf{w}$ is zeromean complex circular symmetric Gaussian noise. We assume that $E\left[\mathbf{w} \mathbf{w}^{\dagger}\right]=\sigma^{2} I_{r}$. The transmitter is constrained in its total power, i.e., $E\left[\mathbf{u}^{\dagger} \mathbf{u}\right] \leq E_{s}$.

We assume that the channel matrix $H$ is known at both ends of the communication system, and that the waveform channel is flat over the bandwidth of interest. The capacity of this channel is known to be (See e.g. [1]):

$$
C=\sum_{i=1}^{n} \log \left(1+\frac{\lambda_{i} P_{i}}{\sigma^{2}}\right) \text { bits } / \text { use }
$$

where $\lambda_{i}$ is the $i$ th eigenvalue of the Hermitian matrix $H H^{\dagger}$, $n=\min \{r, t\}$, and $P_{1}, \ldots, P_{n}$ is the "waterfilling" power allocation with $\sum_{i} P_{i}=E_{s}$.

\section{JOINT OPTIMIZATION}

One can change the values of the eigenvalues $\lambda_{1}, \ldots, \lambda_{n}$ by moving around the transmit/receive antennas. In the first part of this paper we investigate the problem of choosing $\lambda_{1}, \ldots, \lambda_{n}$ so as to maximize the capacity expression in (2).

We consider first the simpler case of line-of-sight channels with far-field approximation. When this is the case, small perturbations of the antenna locations do not change $\sum_{i} \lambda_{i}$ (See example in [2]). In general, $\sum_{i} \lambda_{i}=\sum_{i j}\left|h_{i j}\right|^{2}$, so the constraint on the sum of the eigenvalues corresponds to setting a limit on the total power gain of the channel. Now, the problem becomes that of finding $\lambda_{i}$ 's and $P_{i}$ 's that jointly maximize the capacity under the constraints $\sum_{i} P_{i} \leq E_{s}$ and $\sum_{i} \lambda_{i}=L$ for given $E_{s}$ and $L$. This is a standard maximization problem that we solve using Lagrange multipliers.

From the solution we make the following observations. Without loss of generality assume $\lambda_{1} \geq \lambda_{2} \geq \cdots \geq \lambda_{n}$. When the signal-to-noise ratio $\left(L E_{s}\right) / \sigma^{2}$ is sufficiently small, then the optimal solution requires $\lambda_{1}=L, P_{1}=E_{s}$, and $\lambda_{j}=P_{j}=0$ for all $j>1$. Physically, this corresponds to using the transmit and receive antennas to generate a focused beam. The channel model is then a single channel with maximal signal-to-noise ratio. On the other extreme, when $\left(L E_{s}\right) / \sigma^{2}$ is sufficiently large, then the optimal solution calls for $\lambda_{1}=\cdots=\lambda_{n}=\frac{L}{n}$ and $P_{1}=\cdots=P_{n}=\frac{E_{s}}{n}$. Physically, this corresponds to creating $n$ parallel channels of small signal-to-noise ratio.

\footnotetext{
${ }^{1}$ This work was partially supported by NOKIA/diAx/SBC.
}

\section{PESSIMISTIC CAPACITY}

In the second part of this paper we look at the other extreme: we search for $\lambda_{1}, \ldots, \lambda_{n}$ that minimize the capacity subject to $\sum_{i} P_{i} \leq E_{s}$ and $\sum_{i} \lambda_{i} \geq L$. This corresponds to studying the capacity if an adversary chooses $\lambda_{i}$ 's. In other words, here we do a worst-case analysis when the channel is only assumed to have $\sum_{i} \lambda_{i} \geq L$ but otherwise is as bad as possible. We still maximize over the input power distribution while minimizing over the eigenvalues distribution. We study two possibilities. The first possibility is when the choice of the $\lambda_{i}$ 's is known only at the receiver, so that $\left\{P_{i}\right\}$ cannot depend on $\left\{\lambda_{i}\right\}$. This leads to

$$
C_{p 1}=\max _{\substack{P_{i}: \\ \sum_{i} P_{i} \leq E_{s}}} \min _{\substack{\lambda_{i}: \\ \Sigma_{i} \lambda_{i} \geq L}} \sum_{i=1}^{n} \log \left(1+\frac{\lambda_{i} P_{i}}{\sigma^{2}}\right) .
$$

Solving this optimization problem, we find that the maximizing power distribution should be uniform. This is an intuitive result: if we put much of the power in one channel, the "adversary" can defeat us by setting the gain of that channel to zero. For the eigenvalues, we find that we need only one subchannel, $\lambda_{1}=L$ and $\lambda_{j}=0$ for $j>1$.

The second possibility is when the $\lambda_{i}$ 's are known at both transmitter and receiver. We then find

$$
C_{p 2}=\min _{\substack{\lambda_{i}: \\ \sum_{i} \lambda_{i} \geq L}} \max _{\substack{P_{i}: \\ \sum_{i} \leq P_{s}}} \sum_{i=1}^{n} \log \left(1+\frac{\lambda_{i} P_{i}}{\sigma^{2}}\right) .
$$

One can prove that the minimum is achieved only if all the eigenvalues are nonzero and all the parallel channels are active, i.e., $P_{i}>0 \forall i$. One also finds that the minimum achieving eigenvalues can take at most two possible values. Further more, there is a threshold $T>4(n-1)$ such that if $L E_{s} / \sigma^{2} \leq$ $T$, then the minimum is achieved when $\lambda_{1}=\lambda_{2}=\cdots=\lambda_{n}=$ $\frac{L}{n}$. In the joint optimization problem we found that at low SNR, the best channel is the one that has exactly one nonzero eigenvalue. As one would expect, the worst channel is the one that makes all the eigenvalues equal.

For $L E_{s} / \sigma^{2}>T$ the solution requires that the eigenvalues are all equal except for one dominant eigenvalue. As $L E_{s} / \sigma^{2}$ increases, the dominant eigenvalue grows towards $L$, while the others, strictly positive, decrease towards zero. Again, this is almost the opposite of the result from joint optimization that required $n$ equal strength eigenvalues for high $L E_{s} / \sigma^{2}$.

\section{REFERENCES}

[1] I. Emre Telatar, "Capacity of Multi-antenna Gaussian Channels", AT\&T Bell Laboratories, Internal Tech. Memo., June 1995.

[2] N. Chiurtu and B. Rimoldi, "Varying the Antenna Locations to Optimize the Capacity of Multi-Antenna Gaussian Channels", ICASSP 2000, Istanbul, Turkey. 